\title{
Erratum: Refractive Index of Silicon at $\gamma$ Ray Energies [Phys. Rev. Lett. 108, 184802 (2012)]
}

\author{
D. Habs, M. M. Günther, M. Jentschel, and W. Urban \\ (Received 6 March 2017; published 18 April 2017)
}

DOI: 10.1103/PhysRevLett.118.169904

Herewith, we withdraw our Letter on the measurement of the refractive index of silicon at gamma ray energies. The experimental results suffered from a systematic error induced by diffraction properties of the sample prism. Because of the shutdown of the GAMS5 spectrometer in 2013, a continuation of the research was impossible. Meanwhile, with the commissioning of GAMS6, new measurements were performed. The systematic error has been meanwhile shown to be experimentally reproducible and has been quantitatively included into the evaluation of old data as well as taken into account for new measurements. It was shown that the discovered sign change of the refractive index was not due to refraction but essentially due to an onset of diffraction for energies above a certain threshold and acting with opposite sign. A detailed description of this new evaluation together with a new measurement avoiding this and other minor systematic errors can be found in Ref. [1] and has been submitted to Physical Review. The theoretical interpretation in our Letter explaining the sign change of the real part of the complex refractive index by the contribution of Delbrück scattering also suffered from a calculation error in the numerical integration. This is indicated by recent theoretical work of Donohue [2] and Garanin et al. [3].

[1] M. M. Günther, M. Jentschel, A. J. Pollitt, P. G. Thirolf, and M. Zepf, arXiv:1702.07023.

[2] J. T. Donohue, Phys. Rev. Lett. 110, 129501 (2013).

[3] S. F. Garanin, and E. M. Kravets, Phys. Lett. A 380, 3703 (2016). 